\title{
Euler Polynomials and Combinatoric Convolution Sums of Divisor Functions with Even Indices
}

\author{
Daeyeoul Kim, ${ }^{1}$ Abdelmejid Bayad, ${ }^{2}$ and Joongsoo Park ${ }^{3}$ \\ ${ }^{1}$ National Institute for Mathematical Sciences, Daejeon 305-811, Republic of Korea \\ ${ }^{2}$ Département de Mathématiques, Université d'Evry Val d'Essonne, France \\ ${ }^{3}$ Woosuk University, Samlae, Wanju, Jeonbuk 565-701, Republic of Korea \\ Correspondence should be addressed to Joongsoo Park; jspark0365@gmail.com
}

Received 30 May 2014; Accepted 29 July 2014; Published 27 August 2014

Academic Editor: Elena Litsyn

Copyright (c) 2014 Daeyeoul Kim et al. This is an open access article distributed under the Creative Commons Attribution License, which permits unrestricted use, distribution, and reproduction in any medium, provided the original work is properly cited.

We study combinatoric convolution sums of certain divisor functions involving even indices. We express them as a linear combination of divisor functions and Euler polynomials and obtain identities $D_{2 k}(n)=(1 / 4) \sigma_{2 k+1,0}(n ; 2)-2 \cdot 4^{2 k} \sigma_{2 k+1}(n / 4)-$ $(1 / 2)\left[\sum_{d \mid n, d \equiv 1(4)}\left\{E_{2 k}(d)+E_{2 k}(d-1)\right\}+2^{2 k} \sum_{d \mid n, d \equiv 1(2)} E_{2 k}\left(\left(d+(-1)^{(d-1) / 2}\right) / 2\right)\right], U_{2 k}(p, q)=2^{2 k-2}\left[-((p+q) / 2) E_{2 k}((p+q) / 2+1)+\right.$ $\left.((q-p) / 2) E_{2 k}((q-p) / 2)-E_{2 k}((p+1) / 2)-E_{2 k}((q+1) / 2)+E_{2 k+1}((p+q) / 2+1)-E_{2 k+1}((q-p) / 2)\right]$, and $F_{2 k}(n)=(1 / 2)\left\{\sigma_{2 k+1}^{\dagger}(n)-\sigma_{2 k}^{\dagger}(n)\right\}$. As applications of these identities, we give several concrete interpretations in terms of the procedural modelling method.

\section{Statement of Main Theoretical Results}

Bernoulli polynomials $B_{n}(x)$ and Euler polynomials $E_{n}(x)$ are usually defined by means of the following generating functions: $u e^{u x} /\left(e^{u}-1\right)=\sum_{k=0}^{\infty} B_{k}(x)\left(u^{k} / k !\right)$ and $2 u e^{u x} /\left(e^{u}+\right.$ 1) $=\sum_{k=0}^{\infty} E_{k}(x)\left(u^{k} / k !\right)$.

Bernoulli numbers and Euler numbers are $B_{k}=B_{k}(0)$ and $E_{k}=2{ }^{k} E_{k}(1 / 2)$. We recall the fundamental properties as follows $[1,2]$ :

$$
\begin{gathered}
B_{k}(1-x)=(-1)^{k} B_{k}(x), \quad E_{k}(1-x)=(-1)^{k} E_{k}(x), \\
E_{k}(x)=\frac{2}{k+1}\left\{B_{k+1}(x)-2^{k+1} B_{k+1}\left(\frac{x}{2}\right)\right\}, \\
B_{n+1}(x+1)-B_{n+1}(x)=(n+1) x^{n}, \\
E_{n}(x+1)+E_{n}(x)=2 x^{n}, \\
B_{n}(x+y)=\sum_{k=0}^{n}\left(\begin{array}{l}
n \\
k
\end{array}\right) B_{k}(x) y^{n-k},
\end{gathered}
$$

$$
E_{n}(x+y)=\sum_{k=0}^{n}\left(\begin{array}{l}
n \\
k
\end{array}\right) E_{k}(x) y^{n-k} .
$$

For $n, N \in \mathbb{N}$ and $i, k, l \in \mathbb{Z}$, we define the following:

$$
\begin{aligned}
\sigma_{k}(n) & =\sum_{d \mid n} d^{k}, \\
\sigma_{k, l}(n ; 2) & =\sum_{d \mid n, d \equiv l(2)} d^{k}, \\
\sigma_{k}^{*}(n) & =\sum_{d \mid n, n / d \text { odd }} d^{k}, \\
\sigma_{k}^{\dagger}(n) & =\sum_{d \mid n, n / d \equiv 1(4)} d^{k}-(-1)^{k} \sum_{d \mid n, n / d \equiv-1(4)} d^{k}, \\
\sigma_{k}^{\dagger \dagger}(n) & =\sum_{d \mid n, d \equiv 1(4)} d^{k}-(-1)^{k} \sum_{d \mid n, d \equiv-1(4)} d^{k} .
\end{aligned}
$$

We are motivated by the Besge [3] and Ramanujan [4] identity

$$
\sum_{k=1}^{n-1} \sigma_{1}(k) \sigma_{1}(n-k)=\frac{5}{12} \sigma_{3}(n)+\left(\frac{1}{12}-\frac{1}{2} n\right) \sigma_{1}(n)
$$




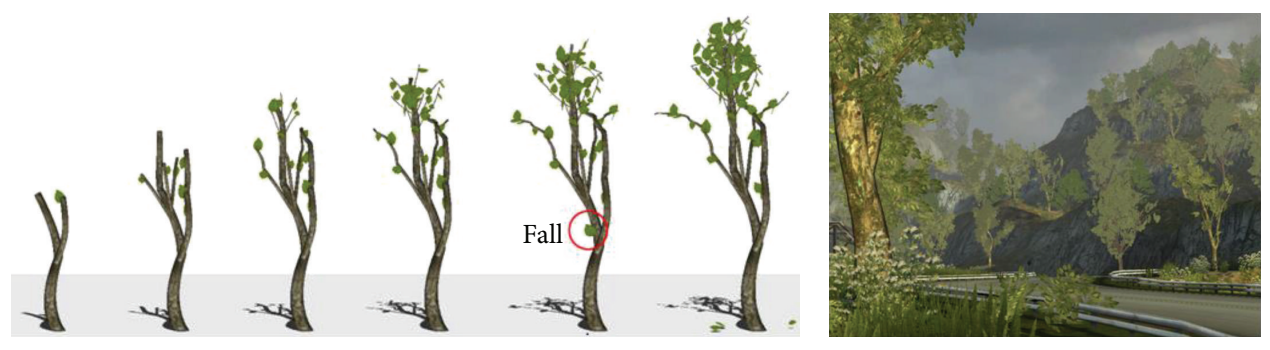

FIGURE 1: MCD tree.

and also by the recent development on the convolution sums. In this paper, we investigate the three combinatoric convolution sums with "even indices." Consider

$$
\begin{aligned}
& D_{2 k}(n):= \sum_{r=0}^{k}\left(\begin{array}{l}
2 k \\
2 r
\end{array}\right) \sum_{m=1}^{n-1} \sigma_{2 k-2 r}^{\dagger \dagger}(m) \sigma_{2 r}^{\dagger \dagger}(n-m), \\
& F_{2 k}(n):=\sum_{r=0}^{k}\left(\begin{array}{l}
2 k \\
2 r
\end{array}\right) \sum_{m=1}^{n-1} \sigma_{2 k-2 r}^{\dagger}(m) \sigma_{2 r}^{\dagger}(n-m), \\
& U_{2 k}(p, q):=\sum_{\substack{a, b, c, d \text { even, } a+b \geq 2 \\
1 \leq l \leq p-1,1 \leq l^{\prime} \leq q-1}}\left(\begin{array}{c}
2 k \\
a, b, c, d
\end{array}\right) \\
& \times \sigma_{a}^{\dagger \dagger}(l) \sigma_{b}^{\dagger \dagger}(p-l) \sigma_{c}^{\dagger \dagger}\left(l^{\prime}\right) \\
& \times \sigma_{d}^{\dagger \dagger}\left(q-l^{\prime}\right),
\end{aligned}
$$

where $\left(\begin{array}{c}2 k \\ a, b, c, d\end{array}\right)=(2 k) ! / a ! b ! c ! d !$ with $a+b+c+d=2 k$. Now, we state our main results.

Theorem 1. For any positive integers $k \geq 1$ and $n \geq 2$, one has

$$
\begin{aligned}
D_{2 k}(n)= & \frac{1}{4} \sigma_{2 k+1,0}(n ; 2)-2 \cdot 4^{2 k} \sigma_{2 k+1}\left(\frac{n}{4}\right) \\
& -\frac{1}{2}\left[\sum_{d \mid n, d \equiv 1(4)}\left\{E_{2 k}(d)+E_{2 k}(d-1)\right\}\right. \\
& \left.+2^{2 k} \sum_{d \mid n, d \equiv 1(2)} E_{2 k}\left(\frac{d+(-1)^{(d-1) / 2}}{2}\right)\right] .
\end{aligned}
$$

Corollary 2. Let $k \geq 4$ be integer and $p$ and $q$ primes with $p \equiv q \equiv-1(\bmod 4)$. Then one has

$$
\begin{aligned}
U_{2 k}(p, q)=2^{2 k-2}[- & \left(\frac{p+q}{2}\right) E_{2 k}\left(\frac{p+q}{2}+1\right) \\
& +\left(\frac{q-p}{2}\right) E_{2 k}\left(\frac{q-p}{2}\right) \\
& -E_{2 k}\left(\frac{p+1}{2}\right)-E_{2 k}\left(\frac{q+1}{2}\right) \\
& +E_{2 k+1}\left(\frac{p+q}{2}+1\right) \\
& \left.-E_{2 k+1}\left(\frac{q-p}{2}\right)\right] .
\end{aligned}
$$

Theorem 3. For any positive integers $k \geq 1$ and $n \geq 2$, one has

$$
F_{2 k}(n)=\frac{1}{2}\left\{\sigma_{2 k+1}^{\dagger}(n)-\sigma_{2 k}^{\dagger}(n)\right\} \text {. }
$$

Theorem 3 can be obtained by $[5,6]$. Therefore, we omit the proof.

\section{Application and Perspective}

2.1. Branch and Leaves Pattern. In real-time virtual system, realistic and efficient generation and expression of plants composing a broad terrain is a continuing problem $[7,8]$. The procedural modelling method using convolution sums of divisor functions (MCD) was suggested for a variety of natural trees in a virtual ecosystem [9]. The basic structure of MCD is that it defines the growth grammar including the branch propagation, a growth pattern of branches and leaves, and a process of growth deformation for various generations of tree. In Figure 1, we illustrate the MCD for the divisor trees.

For more examples see [10]. Theorems 1 and 3 give us basic background for efficient and diverse generations and expressions of trees composing virtual ecosystem or real-time animation processing. In order to apply MCD to the growth structure of a tree model, (5) is modified and expressed in

$$
\sum_{r=0}^{l}\left(\begin{array}{l}
2 l \\
2 r
\end{array}\right) \sum_{k-1}^{N-1} D^{i}\left(B^{i}(x, y) k\right) D^{i}\left(L^{i}(x, y)(N-k)\right)
$$

where $D$ represents various divisor functions, $i$ is the current growth step $\left(g_{s}\right)$, and $N-1$ is the final iteration number of the $i$ th growth step. Here, $D^{i}\left(B^{i}(x, y) k\right)$ is a divisor function that determines the pattern of the number of branches and $D^{i}\left(L^{i}(x, y)(N-k)\right.$ is a divisor function that determines the number of leaves with $l+1$ different types of trees and grasses in the virtual system. Further, $B^{i}(x, y), L^{i}(x, y)$ are the $i$ th exponential function of branches and leaves. Through this model, we verified the possibility of natural and efficient tree growth $[9,11]$. We approximately fix a certain tree model for MCD satisfying the patterns of branch and leaves in virtual system or real tree wood; then using formulae (8), (9), and (10), we obtain the approximate total number for MCD trees leaves by taking two kinds of trees with approximate MCD (Theorem 3), that is, branch pattern $B^{i}(x, y)=\sigma_{0}^{\dagger}$ (resp., $\sigma_{2}^{\dagger}$ ) and leaves pattern $L^{i}(x, y)=\sigma_{2}^{\dagger}$ (resp., $\left.\sigma_{0}^{\dagger}\right)$. We give the first eleven values of the approximate total number $T(n)$ for MCD tree leaves with $n-1$ steps (see Table 1 ). 
TABLE 1

\begin{tabular}{lccccccccccc}
\hline$n$ & 2 & 3 & 4 & 5 & 6 & 7 & 8 & 9 & 10 & 11 & 12 \\
\hline$T(n)$ & 2 & 10 & 24 & 50 & 96 & 148 & 224 & 342 & 452 & 606 & 832 \\
\hline
\end{tabular}

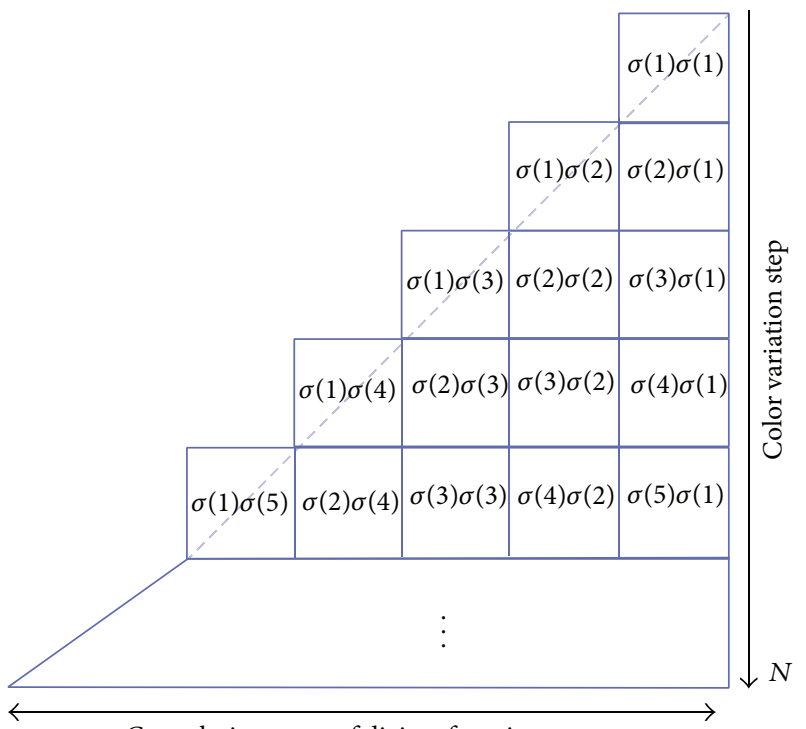

Convolution sums of divisor function
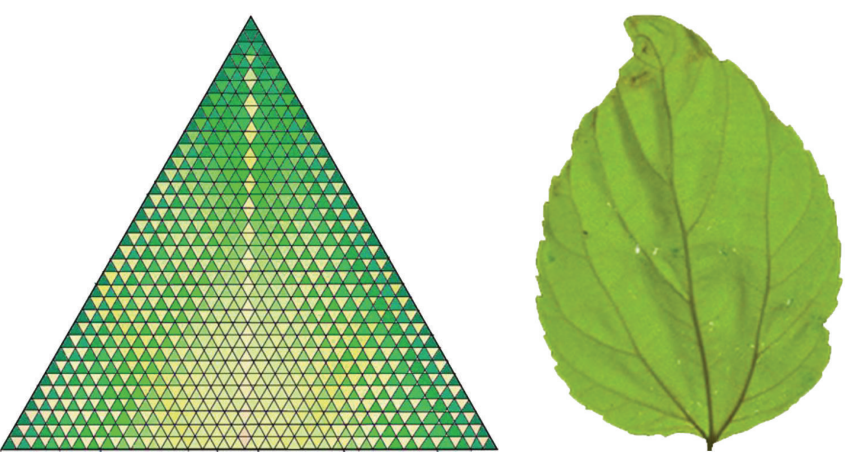

FIgURE 2: Color of leaves.

2.2. Color of Leaves. Leaves are important elements that constitute plants [12]. In [13] we see the leaves model of convolution sum of divisor functions. First, we fix the random color variation step $N$ and then we compute convolution sums by use of (6) as shown in Figure 2 . We denote by $\left(f_{s}, f_{d}\right)$ the start and destination colors; output colors in phase are determined by interpolating convolution sums. Next, based on the minimum and maximum values of the elements of convolution sums of divisor functions, normalized weighted values between 0 and 1 are calculated and then each color table is organized as follows:

$$
\begin{aligned}
t & =\frac{d-d_{\text {min }}}{d_{\text {max }}-d_{\text {min }}}, \\
f & =(1-t) f_{s}+f_{d} t, \\
d_{\text {step }} & =\frac{\left(c_{n}^{\text {max }}-c_{n}^{\text {min }}\right)}{d_{\text {size }}}+1, \\
i d x & =\frac{\left(c_{n}^{i j}-c_{n}^{\text {min }}\right)}{d_{\text {step }}}, \\
c_{\text {leaf }}^{i j} & =c_{d}^{i d x} .
\end{aligned}
$$

The noise map that utilizes Perlin noise is used in the creation of cloud textures for soft color changes. Here, $d_{\text {size }}$ is the number of values that are not duplicated among the elements of convolution sums calculated in the selected table; $c_{n}^{\max }$, $c_{n}^{\text {min }}$ are the maximum and minimum noise values; and $d_{\text {step }}$ indicates the section of noise values influenced by a single element of convolution sums. Once these parameters are fixed, the leaf color is determined by finding the index "idx" and the corresponding color " $c_{d}^{i d x}$ " in the color table comprised of divisor functions that correspond to the noise color in $i$ th row and $j$ th column [13]. Figure 2 represents the image of a color table set created by calculating the color table of each section. Theorem 1 gives the number of values of leaf color and Theorem 3 gives pattern of branch and leaves in the same model. In real-time ecosystem, efficient and fast time is requested. When $n$ is large, the MCD that uses (7) is complicate to compute directly in reasonable time. Our results (8), (9), and (10) give simple formulas for efficient time for them. In the forthcoming papers, we will investigate more applications of real-time virtual ecosystem.

2.3. Proof of Main Results. To prove Theorem 1, we establish Lemmas 4, 6, 7, and 9 and Corollary 5 and we recall Proposition 8.

Lemma 4. Let $k \geq 1$. Set $\widetilde{B}_{k}:=\sum_{j=0}^{k}\left(\begin{array}{c}k \\ j\end{array}\right) 2^{j} B_{j}=2^{k} B_{k}(1 / 2)$. Then one has

$$
B_{2 k+1}\left(\frac{d+1}{2}\right)=\frac{1}{2^{2 k+1}} \sum_{i=0}^{k}\left(\begin{array}{c}
2 k+1 \\
2 i+1
\end{array}\right) \widetilde{B}_{2 k-2 i} d^{2 i+1}
$$

Proof. By formula (4) we obtain

$$
\begin{aligned}
& B_{2 k+1}\left(\frac{1+d}{2}\right)-B_{2 k+1}\left(\frac{1-d}{2}\right) \\
& =\frac{1}{2^{2 k}} \sum_{i=0}^{k}\left(\begin{array}{c}
2 k+1 \\
2 i
\end{array}\right) B_{2 i}\left(\frac{1}{2}\right) 2^{2 i} d^{2 k+1-2 i} .
\end{aligned}
$$

By (15) and (1) we get the lemma. 
By [14, Theorem 3] and Lemma 4 we get the corollary.

Corollary 5. Let $k \geq 1$ and $n \geq 2$. Then

$$
\begin{gathered}
\sum_{s=0}^{k-1}\left(\begin{array}{c}
2 k \\
2 s+1
\end{array}\right) \sum_{m=1}^{n-1} \sigma_{2 k-2 s-1,1}(m ; 2) \sigma_{2 s+1,1}(n-m ; 2) \\
=2^{2 k-1} \sigma_{2 k+1}\left(\frac{n}{2}\right)+\frac{2^{2 k}}{2 k+1} \sum_{\substack{d \mid n \\
\text { dodd }}} B_{2 k+1}\left(\frac{d+1}{2}\right) .
\end{gathered}
$$

Lemma 6. For any integers $\alpha$ and $k \geq 1$, one has

$$
\begin{aligned}
& \sum_{u, v, w \geq 0}\left(\begin{array}{c}
k \\
u, v, w
\end{array}\right)(4 \alpha+1)^{u}(-1)^{w} 4^{v} B_{v}=4^{k} B_{k}(\alpha), \\
& \frac{2}{2 k+1} \sum_{u, v, w \geq 0}\left(\begin{array}{c}
2 k+1 \\
u, 2 v, w
\end{array}\right)(4 \alpha+1)^{u}(-1)^{w} 4^{2 v-1} B_{2 v} \\
& =\frac{2 \cdot 4^{2 k}}{2 k+1} B_{2 k+1}(\alpha)+(4 \alpha)^{2 k} .
\end{aligned}
$$

Proof. Write the series as follows:

$$
\begin{aligned}
\sum_{k=0}^{\infty} \frac{t^{k}}{k !}\left\{\sum_{u+v+w=k}\left(\begin{array}{c}
k \\
u, v, w
\end{array}\right)(4 \alpha+1)^{u}(-1)^{w} 4^{v} B_{v}\right\} \\
=\left(\sum_{u=0}^{\infty}(4 \alpha+1)^{u} \frac{t^{u}}{u !}\right)\left(\sum_{v=0}^{\infty} B_{v} \frac{(4 t)^{v}}{v !}\right) \\
\quad \times\left(\sum_{w=0}^{\infty}(-1)^{w} \frac{t^{w}}{w !}\right) \\
=\left(e^{(4 \alpha+1) t}\right)\left(\frac{4 t}{e^{4 t}-1}\right)\left(e^{-t}\right)=\sum_{k=0}^{\infty} \frac{(4 t)^{k}}{k !} B_{k}(\alpha) \\
=\sum_{k=0}^{\infty} \frac{t^{k}}{k !}\left(4^{k} B_{k}(\alpha)\right) .
\end{aligned}
$$

This completes the proof of (17). On the other hand, (18) may be rewritten as

$$
\begin{aligned}
& \frac{2}{2 k+1} \sum_{u+2 v+w=2 k+1}\left(\begin{array}{c}
2 k+1 \\
u, 2 v, w
\end{array}\right)(4 \alpha+1)^{u}(-1)^{w} 4^{2 v-1} B_{2 v} \\
& =\frac{2}{2 k+1} \\
& \quad \times \sum_{u+v+w=2 k+1}\left(\begin{array}{l}
2 k+1 \\
u, v, w
\end{array}\right)(4 \alpha+1)^{u}(-1)^{w} 4^{v-1} B_{v} \\
& -\frac{2}{2 k+1} \sum_{u+w=2 k}\left(\begin{array}{l}
2 k+1 \\
u, 1, w
\end{array}\right)(4 \alpha+1)^{u}(-1)^{w} 4^{1-1} B_{1} .
\end{aligned}
$$

From (17) we obtain

$$
\begin{aligned}
& \frac{2}{2 k+1} \sum_{u+2 v+w=2 k+1}\left(\begin{array}{c}
2 k+1 \\
u, 2 v, w
\end{array}\right)(4 \alpha+1)^{u}(-1)^{w} 4^{2 v-1} B_{2 v} \\
& =\frac{1}{2(2 k+1)} \sum_{u+v+w=2 k+1}\left(\begin{array}{c}
2 k+1 \\
u, v, w
\end{array}\right)(4 \alpha+1)^{u}(-1)^{w} 4^{v} B_{v} \\
& \quad+\sum_{j=0}^{2 k}\left(\begin{array}{c}
2 k \\
j
\end{array}\right)(-1)^{j}(4 \alpha+1)^{2 k-j} \\
& =\frac{2 \cdot 4^{2 k}}{2 k+1} B_{2 k+1}(\alpha)+(4 \alpha)^{2 k} .
\end{aligned}
$$

This completes the proof of the lemma.

Lemma 7. For any integers $k \geq 1$ and $n \geq 2$, one has

$$
\begin{aligned}
& -\frac{2}{2 k+1} \sum_{u, v, w \geq 0}\left(\begin{array}{c}
2 k+1 \\
u, 2 v, w
\end{array}\right)(-1)^{w} 4^{2 v-1} B_{2 v} \sigma_{u}^{\dagger \dagger}(n) \\
& =-\sum_{\substack{d \mid n \\
d \equiv 1(4)}}\left(\frac{2 \cdot 4^{2 k}}{2 k+1} B_{2 k+1}\left(\frac{d-1}{4}\right)+(d-1)^{2 k}\right) \\
& +\sum_{\substack{d \mid n \\
d \equiv-1(4)}}\left(\frac{2 \cdot 4^{2 k}}{2 k+1} B_{2 k+1}\left(-\frac{d+1}{4}\right)+(d+1)^{2 k}\right) .
\end{aligned}
$$

Proof. We observe that

$$
\begin{aligned}
& -\frac{2}{2 k+1} \sum_{u, v, w \geq 0}\left(\begin{array}{c}
2 k+1 \\
u, 2 v, w
\end{array}\right)(-1)^{w} 4^{2 v-1} B_{2 v} \sigma_{u}^{\dagger \dagger}(n) \\
& =-\frac{2}{2 k+1} \sum_{4 \alpha+1 \mid n}\left(\begin{array}{c}
2 k+1 \\
u, 2 v, w
\end{array}\right)(-1)^{w} 4^{2 v-1} \\
& \quad \times B_{2 v}(4 \alpha+1)^{u} \\
& \quad+\frac{2}{2 k+1} \sum_{4 \alpha-1 \mid n}\left(\begin{array}{c}
2 k+1 \\
u, 2 v, w
\end{array}\right)(-1)^{w} 4^{2 v-1} B_{2 v}(-4 \alpha+1)^{u} .
\end{aligned}
$$

From (18), we get

$$
\begin{aligned}
-\sum_{4 \alpha+1 \mid n} & \left(\frac{2 \cdot 4^{2 k}}{2 k+1} B_{2 k+1}(\alpha)+(4 \alpha)^{2 k}\right) \\
& +\sum_{4 \alpha-1 \mid n}\left(\frac{2 \cdot 4^{2 k}}{2 k+1} B_{2 k+1}(-\alpha)+(4 \alpha)^{2 k}\right) \\
= & -\sum_{d \mid n, d \equiv 1(4)}\left(\frac{2 \cdot 4^{2 k}}{2 k+1} B_{2 k+1}\left(\frac{d-1}{4}\right)+(d-1)^{2 k}\right) \\
& +\sum_{d \mid n, d \equiv-1(4)}\left(\frac{2 \cdot 4^{2 k}}{2 k+1} B_{2 k+1}\left(-\frac{d+1}{4}\right)+(d+1)^{2 k}\right) .
\end{aligned}
$$

We then get lemma. 
We recall Proposition 8 to be modified in Lemma 9.

Proposition 8 (see [15]). Let $N \geq 3,1 \leq i \leq N-1$, and $n, k \in \mathbb{N}$. One has

$$
\begin{aligned}
& \sum_{s=0}^{2 k}\left(\begin{array}{c}
2 k \\
s
\end{array}\right)(-1)^{s} \sum_{m=1}^{n-1} \sigma_{2 k-s}^{b}(m ; i, N) \sigma_{s}^{b}(n-m ; i, N) \\
& =-2 N^{2 k} \sigma_{2 k+1}\left(\frac{n}{N}\right)-\sum_{u=0}^{2 k}\left(\begin{array}{c}
2 k \\
u
\end{array}\right)(-i)^{2 k-u} \sigma_{u}^{b}(n ; i, N) \\
& -\frac{2}{2 k+1} \\
& \quad \times \sum_{u, v, w \in \mathbb{N} \cup\{0\}}\left(\begin{array}{c}
2 k+1 \\
u, 2 v, w
\end{array}\right) N^{2 v-1} B_{2 v}(-i)^{w} \sigma_{u}^{b}(n ; i, N),
\end{aligned}
$$

where $\sigma_{r}^{b}(n ; i, N):=\sum \underset{d \equiv i(N)}{d \mid n} d^{r}-(-1)^{r} \sum_{\substack{d \equiv-i(N) \\ d \mid n}} d^{r}$

Using Lemma 7, we modify Proposition 8 as follows.

Lemma 9. For any $n, k \in \mathbb{N}$ one has

$$
\begin{aligned}
& \sum_{s=0}^{2 k}\left(\begin{array}{c}
2 k \\
s
\end{array}\right)(-1)^{s} \sum_{m=1}^{n-1} \sigma_{2 k-s}^{\dagger \dagger}(m) \sigma_{s}^{\dagger \dagger}(n-m) \\
& =-2 \cdot 4^{2 k} \sigma_{2 k+1}\left(\frac{n}{4}\right)+\frac{2 \cdot 4^{2 k}}{2 k+1} \\
& \quad \times\left(-\sum_{d \mid n, d \equiv 1(4)} B_{2 k+1}\left(\frac{d-1}{4}\right)\right. \\
& \left.+\sum_{d \mid n, d \equiv-1(4)} B_{2 k+1}\left(-\frac{d+1}{4}\right)\right) \\
& +2\left(-\sum_{d \mid n, d \equiv 1(4)}(d-1)^{2 k}+\sum_{d \mid n, d \equiv-1(4)}(d+1)^{2 k}\right)
\end{aligned}
$$

Proof. First, we check that

$$
\begin{aligned}
\sum_{u=0}^{2 k}\left(\begin{array}{c}
2 k \\
u
\end{array}\right)(-1)^{2 k-u} \sigma_{u}^{\dagger \dagger}(n) & \\
= & \sum_{u=0}^{2 k}\left(\begin{array}{c}
2 k \\
u
\end{array}\right)(-1)^{2 k-u} \\
& \times\left(\sum_{d \mid n, d \equiv 1(4)} d^{u}-(-1)^{u} \sum_{d \mid n, d \equiv-1(4)} d^{u}\right) \\
= & \sum_{d \mid n, d \equiv 1}(d-1)^{2 k}-\sum_{d \mid n, d \equiv-1(4)}(d+1)^{2 k} .
\end{aligned}
$$

From Lemma 7 and Proposition 8, our claim follows.
To end the proof of Theorem 1, we observe that

$$
\begin{aligned}
D_{2 k}(n)= & \sum_{s=0}^{2 k}\left(\begin{array}{c}
2 k \\
s
\end{array}\right)(-1)^{s} \sum_{m=1}^{n-1} \sigma_{2 k-s}^{\dagger \dagger}(m) \sigma_{s}^{\dagger \dagger}(n-m) \\
& +\sum_{s=0}^{k-1}\left(\begin{array}{c}
2 k \\
2 s+1
\end{array}\right) \\
& \times \sum_{m=1}^{n-1} \sigma_{2 k-2 s-1,1}(m ; 2) \sigma_{2 s+1,1}(n-m ; 2) .
\end{aligned}
$$

Comparing Corollary 5 and Lemma 9, we see that

$$
\begin{aligned}
D_{2 k}(n)= & \frac{1}{4} \sigma_{2 k+1,0}(n ; 2)-2 \cdot 4^{2 k} \sigma_{2 k+1}\left(\frac{n}{4}\right) \\
& -2 \sum_{d \mid n, d \equiv 1(2)}(-1)^{(d-1) / 2}\left(d-(-1)^{(d-1) / 2}\right)^{2 k} \\
& +\frac{2^{2 k}}{2 k+1} B_{d \mid n, d \equiv 1(4)} B_{2 k+1}\left(\frac{d+1}{2}\right) \\
& +\sum_{d \mid n, d \equiv-1} B_{2 k+1}\left(\frac{d+1}{2}\right) \\
& \quad-2^{2 k+1} \sum_{d \mid n, d \equiv 1} B_{2 k+1}\left(\frac{d-1}{4}\right) \\
& \left.\quad 2^{2 k+1} \sum_{d \mid n, d \equiv-1(4)} B_{2 k+1}\left(\frac{d+5}{4}\right)\right]
\end{aligned}
$$

From (3) we have

$$
\begin{aligned}
& D_{2 k}(n) \\
& =\frac{1}{4} \sigma_{2 k+1,0}(n ; 2)-2 \cdot 4^{2 k} \sigma_{2 k+1}\left(\frac{n}{4}\right) \\
& \quad-2 \sum_{d \mid n, d \equiv 1(2)}(-1)^{(d-1) / 2}\left(d-(-1)^{(d-1) / 2}\right)^{2 k} \\
& +\frac{2^{2 k}}{2 k+1}\left\{B_{2 k+1}\left(\frac{d-1}{2}\right)-2^{2 k+1} B_{2 k+1}\left(\frac{d-1}{4}\right)\right\} \\
& \quad \times \sum_{d \mid n, d \equiv 1(4)}\left\{B_{2 k+1}\left(\frac{d+1}{2}\right)-2^{2 k+1} B_{2 k+1}\left(\frac{d+1}{4}\right)\right\}
\end{aligned}
$$




$$
\begin{aligned}
& +\sum_{d \mid n, d \equiv 1(4)}(2 k+1)\left(\frac{d-1}{2}\right)^{2 k} \\
& -\sum_{d \mid n, d \equiv-1} 2(4)
\end{aligned}
$$

By (2) and (3) we get the theorem.

Proof of Corollary 2. From ([16, page 150]) we can derive

$$
\begin{aligned}
\sum_{r=1}^{k-1}\left(\begin{array}{c}
2 k \\
2 r
\end{array}\right) E_{2 r}(x) E_{2 k-2 r}(y) & \\
= & (1-x-y) E_{2 k}(x+y)+E_{2 k+1}(x+y) \\
& +(y-x) E_{2 k}(y-x)-E_{2 k+1}(y-x) \\
& -E_{2 k}(x)-E_{2 k}(y) .
\end{aligned}
$$

We set $x=(p+1) / 2$ and $y=(q+1) / 2$ in (31) and get the corollary.

\section{Conflict of Interests}

The authors declare that there is no conflict of interests regarding the publication of this paper.

\section{References}

[1] A. Erdelyi, Higher Transcendental Functions, vol. 1, McGraw Hill, New York, NY, USA, 1953.

[2] H. M. Srivastava and A. Pinter, "Remarks on some relationships between the Bernoulli and Euler polynomials," Applied Mathematics Letters, vol. 17, no. 4, pp. 375-380, 2004.

[3] M. Besge, "Extrait d'une lettre de M. Besge à M. Liouville," Journal de Mathématiques Pures et Appliquées, vol. 7, p. 256, 1862.

[4] S. Ramanujan, "On certain arithmetical functions," Transactions of the Cambridge Philosophical Society, vol. 22, pp. 159-184, 1916.

[5] B. Cho, D. Kim, and H. Park, "Evaluation of a certain combinatorial convolution sum in higher level cases," Journal of Mathematical Analysis and Applications, vol. 406, no. 1, pp. 203210, 2013.

[6] D. Kim and A. Bayad, "Convolution identities for twisted Eisenstein series and twisted divisor functions," Fixed Point Theory and Applications, vol. 2013, article 81, 2013.

[7] W. Palubicki, K. Horel, S. Longay et al., "Self-organizing tree models for image synthesis," ACM Transactions on Graphics, vol. 28, no. 3, article 58, 2009.

[8] P. Tan, T. Fang, J. Xiao, P. Zhao, and L. Quan, "Single image tree modeling," ACM Transactions on Graphics, vol. 27, no. 5, article 108,2008 .

[9] J. Kim, D. Kim, and H. Cho, "Procedural modeling of trees based on convolution sums of divisor functions for real-time virtual ecosystems," Computer Animation and Virtual Worlds, vol. 24, no. 3-4, pp. 237-246, 2013.

[10] http://open.nims.re.kr/JrDr/.
[11] J. Kim, D. Kim, and H. Cho, "Tree growth model design for realistic game landscape production (Korean)," Journal of Korea Game Society, vol. 13, no. 2, pp. 49-58, 2013.

[12] H. Xiao and X. Chen, "Modeling and simulation of curled dry leaves," Soft Matter, vol. 7, no. 22, pp. 10794-10802, 2011.

[13] J. Kim and D. Kim, "The procedural modeling and visualization of multiple leaves," Submitted.

[14] D. Kim and Y. K. Park, "Bernoulli identities and combinatoric convolution sums with odd divisor functions," Abstract and Applied Analysis, vol. 2014, Article ID 890973, 8 pages, 2014.

[15] B. Cho, D. Kim, and H. Park, "Certain combinatorial convolu tion sums for divisor functions and Bernoulli numbers," accepted to Bulletin of the Korean Mathematical Society.

[16] W. Chu and R. R. Zhou, "Convolution of Bernoulli and Euler polynomials," Sarajevo Journal of Mathematics, vol. 6, no. 18, pp. 147-163, 2010. 


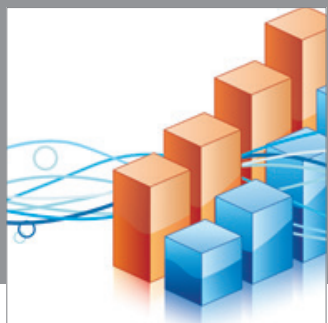

Advances in

Operations Research

mansans

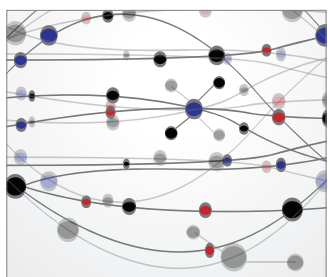

The Scientific World Journal
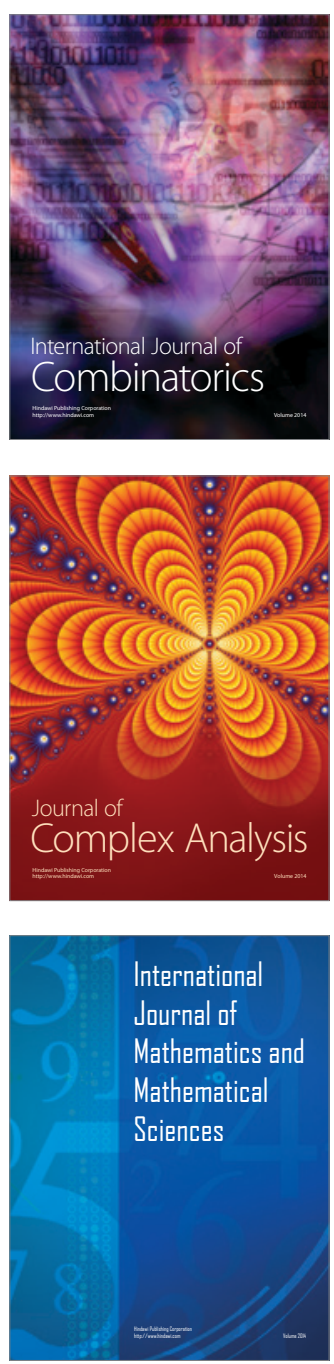
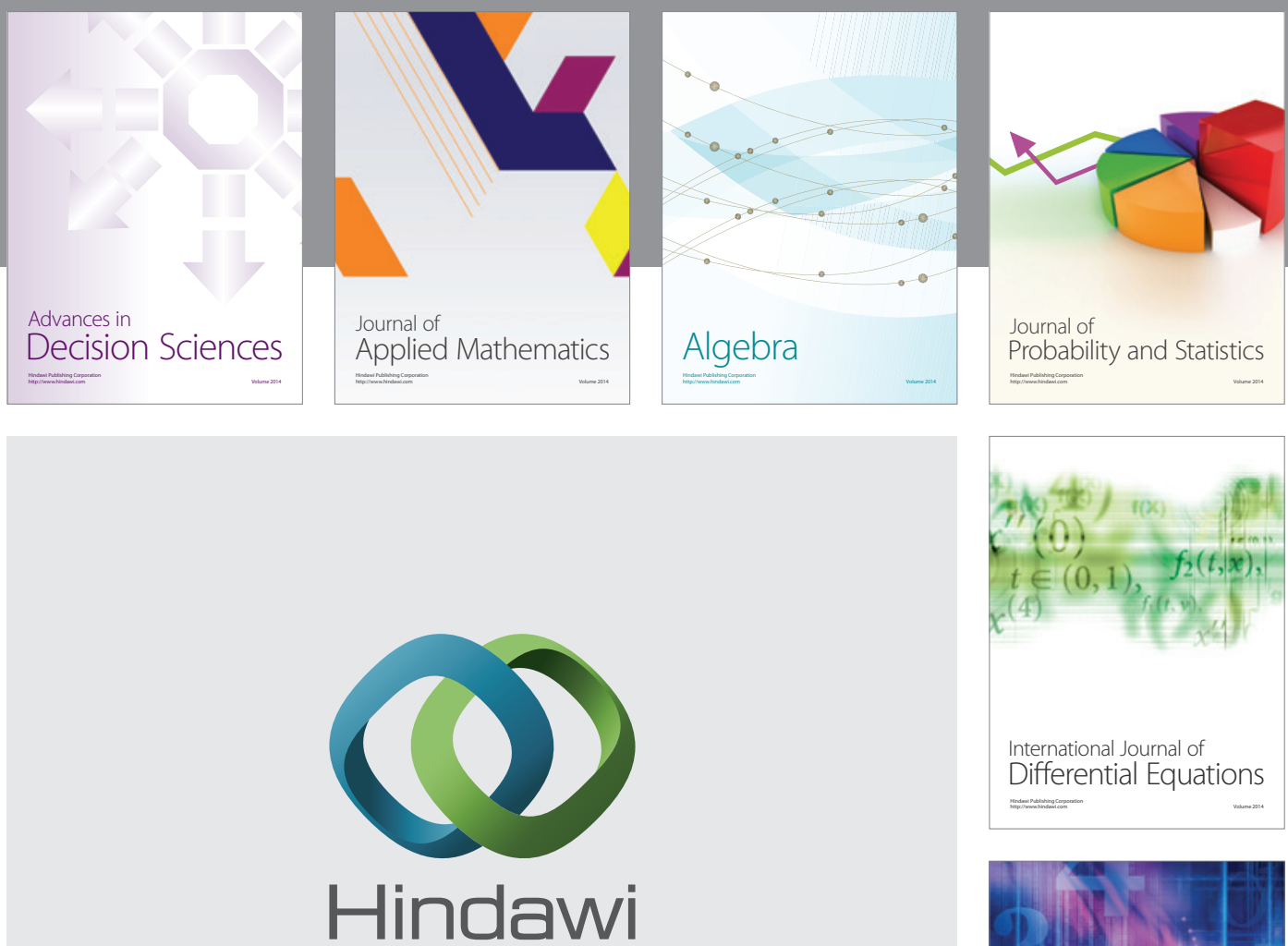

Submit your manuscripts at http://www.hindawi.com
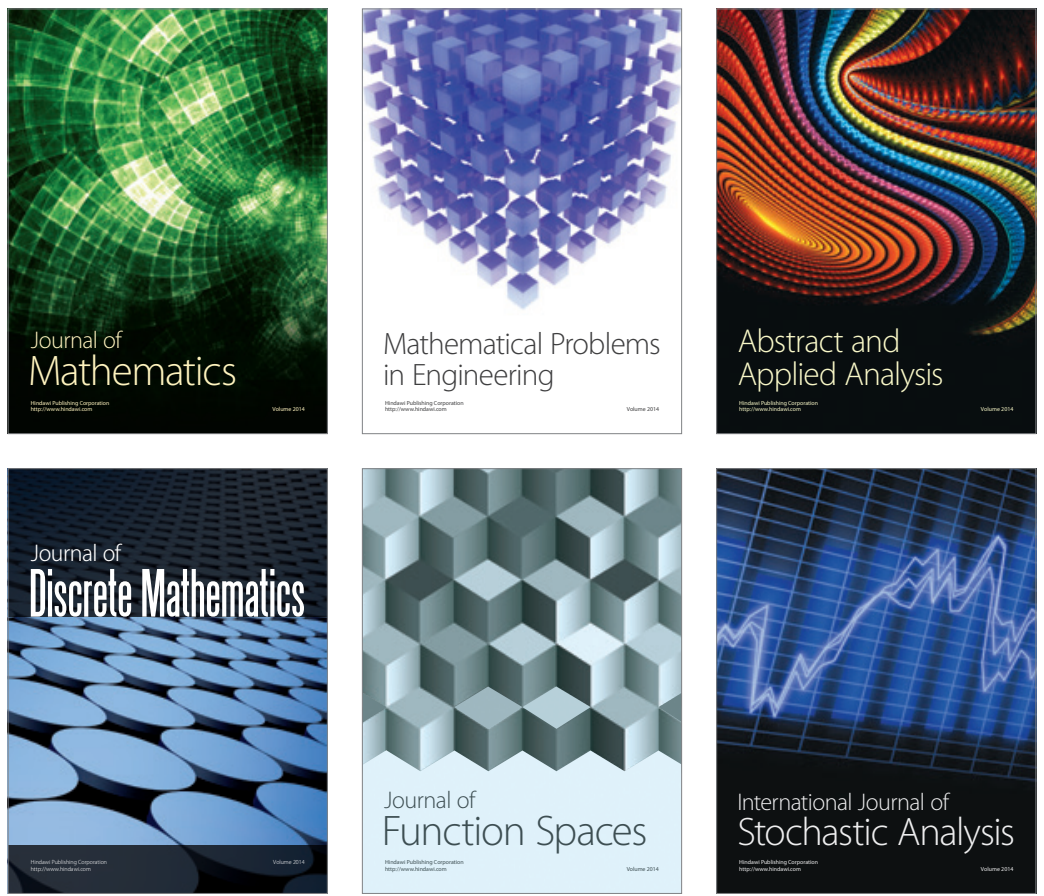

Journal of

Function Spaces

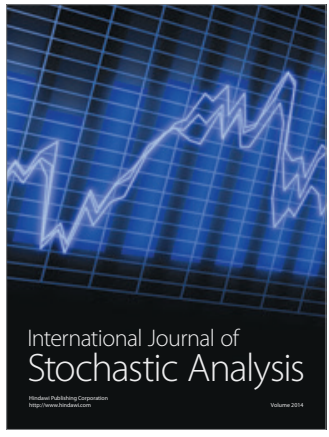

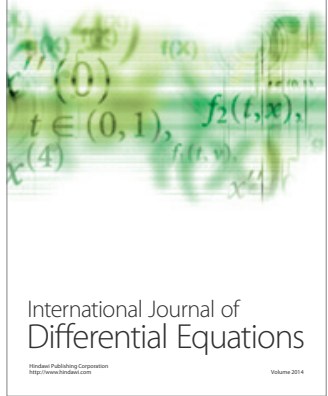
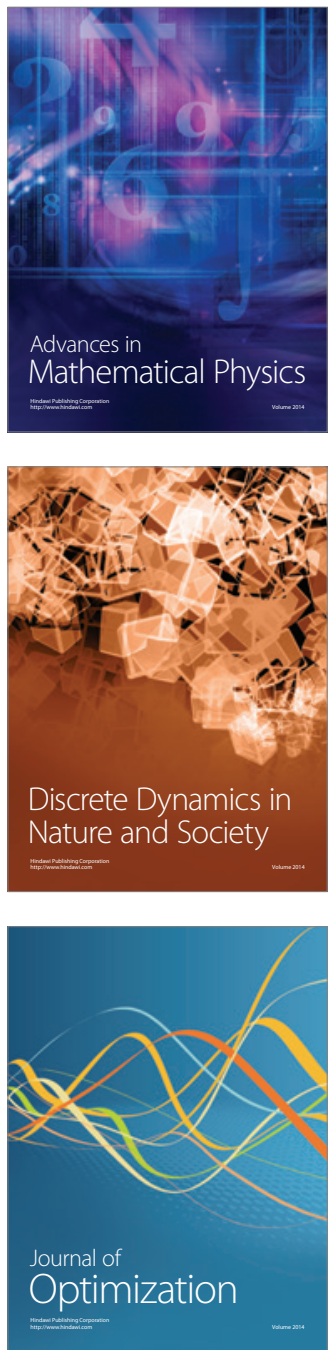